\title{
Misión actual de la Compañía de Jesús
}

\author{
Ignacio Ellacuría
}

Nola de la redacción

Hace diez años, en 1983, los jesuitas celebraron su Congregación General XXXIII. Para mejor prepararla desde Centroamérica el P. Ignacio Ellacuría tuvo la idea de escribir un documenso que, eventualmente, pudiera servir de base a una declaración fundamental que reorientase la misión de la Compañia de Jesús recalcando y profundizando el decreto cuarto de la anterior Congregación General XXXII; la misión de la Compañía consisse en "la defensa de la fe y la promoción de la justicia". La idea fundamensal del documento era que la conversión de la universal Compañía al mundo de los pobres. especialmente a ese mundo de los pobres que es el tercer Mmundo. darla a todo el cuerpo de la Compañía y a sus miembros una nueva vitalidad aposiólica y religiosa.

Publicamos ahora dicho documento anse todo, porque, aunque en él colaboraron varios jesuitas centroamericanos, tanto la inspiración como la mayor parte del texto son abra del $P$. Ellacuría; se trata, pues, de publicar otro de sus escritos inéditos. Pero, ademós, porque su contenido sigue siendo de gran actualidad para todos y especílicamente para los jesuitos, ya que $\rightarrow$ por coincidencia- este año de 1993 se va a convocar la XXXIV Congregación General. He aquí el texio del P. Ellacuría.

$$
\text { * } \pitchfork
$$

1. San Ignacio de Loyola buscaba siempre y renovadamente encontrar la voluntad de Dios según los tiempos, los lugares y las personas. Los Ejercicios espirituales no son tanto un mélodo para conocer especulativamente la esencia divina, sino para conocer a Jesús y su llamada en orden a una acción. Suponen que no se sabe de antemano cual es la volumtad de Dios sobre aspectos esencia- 
les de la vida personal y de la vida institucional y que, por tanto, hay que buscarla y encontrarla. Respecto de la propia Compañía de Jesús no está tampoco determinado de una vcz por todas cuál es el modo concreto de realizar lo que formalmente puede determinarse corno promoción y defensa de la fe, servicio a la lglesia, etc.

Hay, por otra parte, momentos decisivos en la hístoria de los hombres y en la historia de la Iglesia, en los que se necesitan nuevos planteamientos y decisiones, quc pucden suponer novedades importanles para los jesuitas en particular y para la Compañia en general. Esos momentos nuevos vienen caracterizados por distintos acontecimientos históricos, que son a la vez manifestación y causa de grandcs cambios sociales y, o eclesiásticos Tal fue el caso de la reforma protescante, la renovación humanista del renacimiento, el descubrimiento y colonización de grandes tcrritorios del mundo por diversos países europeos, la ilusiración y la revolución francesa, el desaurollo galopante de la ciencia y la tecnología, el urbanismo, las revoluciones marxistas, etc.

Como uno de esos momentos cruciales ha de verse la aparición del tercer mundo en contraposición de los ouros dos mundos, de los cuales en parte es su resultado, pero frente a los cuales mantiene su peculiaridad fundada en una riqueza propia de índole espiritual y cultural. Una profunda visión cristiana e ignaciana sobrc este mundo podría reorientar parte importante de la misión de la Compañía en nuestros días.

2. Para determinar lo que debe hacer hoy la Compañia de Jesús, debiera toda ella, y en este momento la Congregación General, situarse en lo que san Ignacio puso como primer preámbulo de la contemplación de la encamación: mirar cómo ven las tres divinas personas el mundo de hoy, una vez que el Hijo nos ha hecho conocer al Padre y ha enviado a su Espírilu; hay que intentar ver cómo ve el Padre nuestro mundo desde su patemidad, cómo lo ve el Hijo como salvador de los hombres y anunciador del reino, cómo lo ve el Espíritu Santo como dador de vida.

Visto este mundo con ojos trinitarios aparecen en él muchas historias personales, wodas distintas, cada una con sus propios problemas, ilusiones y esperanzas, sufrimientos y angustias, proyectos de vida. Pero aparecen también las vidas personales fuertemente condicionadas por unos determinados procesos sociales e históricos no queridos por el Padre nuestro que está en los cielos para sus hijos; unos procesos sociales e históricos que constituyen en su conjunto un verdadero pecado social porque son resultado de comportamientos objetivamente pecaminosos, constituyen un principio fundamental de injusticia y, sobre todo, niegan la voluntad y la presencia de Dios entre los hombres.

Efectivamente, unos pocos pueblos con población relativamente pequefla

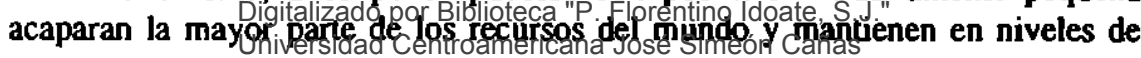


vida infrahumana a otros muchos pueblos mediante formas múltiples de violencia. Son pueblos, muchos de los cuales se dicen pertenecer a la civilización cristiana. que han hecho y siguen haciendo coda suerte de violencia, unas veces para conquistar y someter a los otros pueblos y otras para que no salgan de su órbica de dominación. Son pueblos que han hecho y siguen haciendo violencia económica, explotando indirectamente el trabajo de otros pucblos así como los recursos de sus materias primas. Son pueblos que ejercen una violencia cultural despojando a los otros pueblos de sus formas más profundas y propias de ver el mundo, de organizar la sociedad, de establecer sus propios valores. Todo ello va en menoscabo de la identidad de esos pueblos, de su derecho a la plena autodeterminación, de su soberanía nacional, pero sobre todo en detrimento de su derecho fundamental a la vida que se ve amenazada y aun destruida por el hambre, por la enfermedad, por la miseria, por falta de educación y no en pocos casos por las formas más violentas de represión. La Trinidad no pucde menos de ver en todo esto una de las formas más palpables y más graves del pecado del mundo.

Y frente a estos pueblos, que agrupando a un reducido número de la población mundial acaparan la mayor parte de los recursos de la humanidad y llevan unos niveles de vida escandalosamente distantes de los niveles infra-humanos que tiene que soportar en relación con ellos la mayorfa de los hombres, están esos otros pueblos que agrupan la mayor parte de la población mundial. Unos pueblos que son vistos por la Trinidad con ojos de amor y amados y comprendidos con infínita misericordia como los hijos más queridos, que necesitan tener vida y vida en abundancia. Conssituyen la mayor parte de la humanidad, son los más pobres e injustamente tratados, que han sido violentamente apartados de los bienes más necesarios de este mundo para que unos pocos disfnuten hasta la saciedad de los bienes más superfluos. Son, por ouro lado, los pueblos más religiosos que sienten mayor necesidad de Dios porque en los hombres no ven posibilidad de ayuda; son también pueblos que esłán en las condiciones históricas más consonantes con el evangelio, con lo que fue la condición de vida del propio Hijo de Dios y con lo que es su mensaje de salvación. En esos pueblos, la fe cristiana no tiene que encontrar sofisticadas explicaciones espiritualizantes para cobrar came histórica como la cobró en el Jesús histórico que vivió pobre entre uno de esos pueblos pobres, sometido también y explotado por el pueblo romano dominante, aliado con los grupos sociales nacionales a los que Jesús critico y condenó tan severamente.

Frente a exta situación de contraste, la respuesta divina no es el arrepentimiento por haber creado un mundo en que haya sido posible esta situación, sino un inmenso deseo de salvar al genero humano, especialmente a aquellos que han sido tan duramente castigados por la historia y por el pecado que se ha ido apoderando cada vez más del mundo. La Companta de Jesús tiene también que ver el mundo desde esta perspectiva trinitaria, que no hace distinciones entre lo religioso y lo profono porque todo el mundo y hodo en el mundo es creación de Universidad Centroamericana José Simeón Cañas 
Dios y lodo el mundo y lodo en el mundo debe ser salvado según su propia naturaleza y según la voluntad divina. La distinción fundamental es entre el bien y el mal, entre la gracia y el pecado, entre la vida y la muerte: la vida, el bien, la gracia deben ser multiplicados, mientras que la muerte, el mal y el pecado deben ser abolidos.

3. Para lograr esto ha enviado el Padre a su Hijo bien amado y al Espíritu que da vida. Por el Hijo sabemos lo que es la voluntad del Padre y por el Espíritu podemos escrutar esa voluntad entre los signos de los tiempos y los aconteceres de la historia. El "Rey etemal" se ha puesto frente a todos los hombres y frente al universo mundo y todos los enemigos para su llamada de conquistar el mundo y así entrar en la gloria de su Padre. Pero no se trala de una marcha triunfal como la que pretenden los que han venido a dominar y no a servir. Por eso avisa que quienes lo quieren acompafiar y seguir en la construcción de su reino han de trabajar con él y como él para que ese mundo en pecado se salve, para que ese mundo dividido se reconcilie y llegue a constituirse en fiel expresión del reino de Dios, reino del Padre.

Por eso quiere que, como él, sus seguidores se encarnen plenamente entre los marginados del mundo para que lleguen a ser pueblo de Dios aquellos a los que ni siquiera se les ha dejado llegar a ser pueblo; quiere que se hagan como esos más pequeños para realmente hacerse uno de ellos de modo que desde ellos prediquen y realicen la salvación y divinización que el Padre quiere para todos sus hijos. Es desde la cruz desde donde se realiza la salvación y la resurrección a una nueva vida. Así la misión permanente de los seguidores de Jesús, especialmente de aquellos que "más" se quieren señalar en su servicio, cobra nuevas características misionales.

a) Es necesario hacer eficazmente una opción preferencial por los pobres de modo que la opción general por la pobreza adquiera un realismo nuevo. Frente a la inmensa mayoría del mundo pobre, carece de sentido vivir como ricos y para los ricos. El seguimiento de Jesús pobre lleva al acompañamiento de los hombres pobres. A los pueblos del tercer mundo y a los que en toda la tierra son pobres, marginados y explotados, hay que hacerles llegar la buena nueva de la liberación. Son las víctimas inocentes de la historia de la cristiandad y no del cristianismo, de la cristiandad que hizo de la riqueza y del poder los aliados naturales de la Iglesia. Son también las victimas de la anti-cristiandad, que no del anti-cristianismo, una anti-cristiandad que intentó imponer por la fuerza los derechos de los desposeídos, pero haciendo del monopolio del poder y de la riqueza arma necesaria de expansión y sojuzgamiento. A esas víctimas especialmente hay que dirigirles la buena nueva para liberarlas de sus cadenas, abrir sus ojos, ayudarlas a caminar, a convertirse en sujetos de su propio presente y de su futuro; una buena nueva que es válida para convertir los corazones, pero que es también poderosa para transformar la historia, si es que es asumida plenamente por los pugledpsaydas ogganizaciones pepulanesate, S.J." 
b) Esa palabra de salvación debe ir dirigida a superar la violencia de la que es victima la mayor parte de la humanidad. Dios Padre en su Hijo por el Espíritu de amor que ha enviado sobre el mundo dice un no rotundo a esa violencia fundamental y originaria que hacen los poderes de este mundo contra sus hijos más desvalidos, privándolos hasta de la vida, si es necesario, para mantener injusticias estructurales que van en beneficio de unos pocos y en maleficio de la mayor parte de la humanidad.

Esa violencia debe, por tanto, ser analizada, descubierta, denunciada y superads. Debe ser superada por los pueblos que la sufren, no movidos por odio o resentimiento sino por amor incluso a los enemigos, sin que esto excluya el uso de la fuerza en la medida que este uso produzca mayores bienes que males en orden a que los hombres y los pueblos tengan mayor vida. Son múltiples y sutiles los mecanismos mediante los cuales se hace violencia a los más débiles y por eso quienes predican la libertad y el amor de los hijos de Dios, los que tienen sed de justicia, deben esforzarse por desenmascararlos y superarlos, por más resistencia que encuentren. Tarea nada fácil que llevará consigo trabajo y persecución, ser tenidos por locos de cara.a los poderes de este mundo, cuando no ser tenido por enemigos de la civilización cristiana.

c) Como parte de la superación de esta violencia fundamental y radical está, por un lado, la superación de los nacionalismos que so pretexto de la seguridad nacional someten los derechos humanos y las autonomías de los pueblos con formas intolerables de ingerencia y dominación. Por otro lado está la superación del enfrentamiento de los sistemas imperialistas hoy actuantes, que son formas idolátricas de vida, de cultura y también de acción políbica, económica y militar. Son los pueblos del tercer mundo quienes deben evitar caer en el ámbito ideológico y estructural de los dos extremos del capitalismo hoy imperante y del colectivismo hoy efectivo. Y esto mediante una solidaridad cada vez más plena de los pueblos que han sufrido los males de la lucha hegemónica de los dos imperios, que hoy se disputan el dominio de la humanidad.

d) Visto positivamente, el mensaje de la salvación debe promover la puesca en marcha de una civilización de la pobreza más afín con lo que es la fe cristiana y más afin con lo que es la realidad del hombre y lo que es la relación "recursos mundiales-bienestar universal". El tercer mundo, no en cuanto sojuzgado y dominado, sino en cuanto realidad objetiva se puede convertir en el lugar natural de la fe cristiana, que desde luego no tiene su lugar entre las naciones más ricas y dominantes de la tierra.

La pobreza es una necesidad histórica y no sólo un consejo de perfección en el momento actual desde una perspectiva mundial y lo será aún por muchos a hos en beneficio de formas más altas de humanidad. Una pobreza que en gran parte es resultado de la explotación, pero que puede asumirse activa y voluntariamente como ung aproyechamiento y distribución de los bienes de la tierra 
que haga posible el que todos tengan acceso a unos medios materiales y culturales que permitan tener una vida verdaderamente humana. Esa pobreza es la que realmente da espacio al espíritu, que ya no se verá ahogado por el ansia de tener más que el otro, por el ansia concupiscente de tener toda suerte de superfluidades, cuando a la mayor parte de la humanidad le falta lo más necesario.

Podrá entonces florecer el espíritu, la inmensa riqueza espiritual y humana de los pobres y los pueblos del tercer mundo, hoy ahogada por la miseria y por la imposición de modelos culturales más desarrollados en algunos aspectos, pero no por eso más plenamente humanos. Se vivirá así más fácilmente el espiritu evangélico, según el cual no hace falta tener mucho para ser mucho, antes al contrario hay un límite en el que el tener se opone al ser tanto en el caso de la máxima privación como en el caso de la superfluidad; no habrá lugar para la avaricia, que mantiene a los hombres en tensiones de trabajo insufribles sólo para amontonar riquezas en las que poner la propia seguridad y la de los suyos 0 , lo que es peor, con las que poder comprar las voluntades y la moral de los demás.

Se entraría así en una civilización de la pobreza plenamente coherente con la predicación de Jesús, una civilización de la austeridad, del compartir, de la comunicación de bienes y de vidas, de la creatividad humana como florecimiento de la gracia interior; una civilización, abierta a lo transcendente y muy en especial a la forma cristiana de la transcendencia tal como ésta se revela en Jesús de Nazaret que se igualó con los más pobres para hacer ver de modo nuevo la gloria de Dios. Virtudes esenciales como la abnegación, la donación de sí mismo y de las cosas propias, la esperanza en Dios, la humildad, el amor, etc., tendrían en esta civilización de la pobreza el terreno abonado para que fructificase ciento por ciento la semilla sembrada abundantemente por los seguidores de Jesús.

e) La propia fe cristiana inculturizándose en este suelo nutricio de la civilización de la pobreza, en el que primeramente dio sus frutos y en el que ha seguido dándolos a lo largo de la historia podría aportar mucho a que esa civilización de la pobreza fuera impregnada de vida cristiana. Hay una consonancia fundamental entre lo más hondo de la fe cristiana desde la perspectiva de las bienaventuranzas y del sermón del monte con lo que son las necesidades históricas de nuestro mundo.

En el tercer mundo están dadas las condiciones objetivas para vivir plenamente el mensaje cristiano y este tercer mundo, escándalo de los otros dos, podría convertirse en su salvador, cuando a esas condiciones objetivas añadiese los valores explícitos de la conciencia cristiana. Pero, ¿cómo los va a tener si no se los anuncia? Y ¿cómo se van a anunciar si no llegan los mensajeros de la $\mathrm{fe}$ ? Por eso el Rey etemal repite y renueva su llamado para que sus más cercanos seguidores, aquellos que de verdad quieran ser̂alarse en su seryicio, se dediquen Universidad Centroamericana José Simeón Cañas 
preferencialmente a esta concreción del mundo de los pobres que es el tercer mundo.

Ya los países de América Latina se dibujan como la gran reserva de la fe cristiana para el siglo veintiuno. Hay que pensar y vivir la fe y las estructuras eclesiales desde la realidad histórica de América Latina donde se esconde históricamente el futuro de la Iglesia, pero sin olvidar por ello a todos los pobres de la tierra $y$, muy en especial, a las grandes poblaciones que constituyen el tercer mundo. No es en los países ricos donde la fe cristiana puede dar de sí lo que es en sí y sería un error que cn nombre de la fe cristiana se impulsase a los países pobres y jóvenes hacia los modelos de desarrollo y civilización que propugnan los paiscs y los sistemas que han hecho de la civilización de la riqueza el modelo de su desarrollo. Pero no por eso los países ricos se verían privados de la fe; sólo que el centro del cristianismo no estaría ya en ellos, sino donde ahora es su lugar (sobre) natural, que no es tanto donde está el peso de la institucionalidad, sino donde está el peso de la mayoría de los creyentes y el peso de los más preferidos por el Padre en Jesús. Desde este nuevo lugar histórico de la pobreza vendría la interpelación a los países y pueblos ricos para que dejadas sus riquezas siguiesen a Jesús.

5. La Compañia de Jesús debe aprestarse de nuevo a responder generosamente, como avanzada de la Iglesia, al llamado que hace el Padre en el Hijo, confiada en la abundante presencia viva y creadora del Espíritu Santo dentro de la Iglesia. Se trata de que escuche la llamada y encuentre los caminos nuevos de responder a ella. La Compañía como institución que está instalada en países del primer mundo, pero que también vive y se enraíza en países del tercer mundo, tiene que hacer un nuevo esfuerzo para establecer como punto de referencia histórico lundamental ese gran pueblo del mundo sacrificado, que ha sido despojado por hombres y pueblos violentos y ante el que los sacerdotes y escribas pasan con frecuencia de largo ocupados en sus cosas espirituales o culturales con el pretexto de que son más altas y divinas.

Todo el espíritu de los ejercicios que reclama el ponerse con Jesús pobre, humilde y ofendido debe encarnarse ahora en ponerse efectivamente con la prolongación de ese Jesús pobre, humilde y ofendido, que es la mayor parte de la humanidad que vive en el tercer mundo hambrienta, sedienta, desnuda y en cárcel, entre la que Jesús prometió estar de manera especial con tanta o mayor explicitación de lo que prometió estar en la eucaristía. La Compañía, como la Iglesia, son para el mundo y dentro de ese mundo no sólo ni fundarnentalmente para unas elites, sino para las inmensas mayorias de pobres y crucificados. La Iglesia es para que el reino de Dios llegue a ser, para que la voluntad de Dios, salvífica y humanizante, se haga realidad en la historia de los hombres. Ese ser para el reino no para sí es el vaciamiento especílico exigido a la Iglesia y a la Compañía y el primer paso para la conversión, que la criscianizará, la rehará Digitalizado por Biblioteca "P. Florentino Idoate, S.J."

Universidad Centroamericana José Simeón Cañas 
siempre evangélicamente en su misión y en su identidad. Ese ser para el mundo es también el talante fundamental de la Companía que le hará escuchar y cumplir la voluntad de Dios hoy sobre el mundo para que esa voluntad se haga realidad.

a) Punto absolutamente esencial de esa misión hoy es defender y propiciar la vida amenazada y aniquilada en gran parte de la humanidad especialmente en el tercer mundo, donde esa anulación de la vida no es un metáfora sino una realidad. Ciertamente no es esa toda su misión, pero sí aquella sin la cual las otras misiones no serán plenamente teologales, pues no verificarán la fe en un Dios creador, salvador, ni serán creíbles por una humanidad amenzada de muerte. De esa forma se historizarán para nuestro tiempo los fundamentos trascendentes de la realidad: la fe en un Dios, creador y padre de todos, defensor de las mayorías pobres; la fe en un único Señor, anunciador e iniciador de una buena noticia a los pobres, muerto y resucilado por esa buena noticia; la fe en un único Espírilu, Señor y dador de vida, que nos re-descubre siempre la verdad del mundo y de Dios y que consuela a todos aquellos que están privados de vida.

Una fe así historizada hace santa a la Iglesia, la asemeja a su Señor Jesús porque defiende la verdad, es movida realmente por el amor, tiene entrañas de misericordia para compadecerse de las multitudes, pone a la Iglesia en su verdadero lugar, el mundo de los pobres y de los crucificados, es perseguida y atacada por los ídolos de la muerte. Una fe así historizada hace que las iglesias locales se lleven mutuamente, den y reciban cada una lo mejor que tienen de fe, esperanza, amor, creatividad pastoral, litúrgica y teológica, recursos materiales.

La comunión en el problema más grave de la humanidad y la corresponsabilidad ante ese problema pone las bases para la Iglesia una. Los diversos aportes para que haya vida y vida en plenitud enriquecen a la Iglesia universal. El llevarse solidariamente las iglesias locales construye realmente el único pueblo de Dios, cuerpo de Cristo y templo del Espíritu. Pero ese llamado a la verdadera catolicidad y al verdadero ecumenismo tiene su centro -que cambia históricamente- allá donde las iglesias locales se han encarnado en las cruces de la historia y han trabajado por bajar de la cruz a pueblos crucificados. Es hoy un privilegio y una responsabilidad de las iglesias del tercer mundo haber desencadenado esa catolicidad.

La Compañía, fiel a su carisma, debe estar en la avanzada que, en el mundo actual, significa estar en el más abajo de la historia junto a los crucificados. Desde esas cruces debe denunciar todo aquello que en su contomo inmediato contribuye a crear la miseria y el dolor del mundo, debe compartir la suerte de aquellos para quiene vivir es su máxima tarea y la muerte su destino más cercano; debe defender los derechos de los hijos de Dios, sobre todo el derecho a la vida. A la Compañía le toca hoy algo aparentemente mínimo, pero que es en verdad máximo alos oios de Dios escondido en el mundo de los pobres: la vida Universidad Centroamericana José Simeón Cañas 
de los pobres, la libertad del pueblo pobre de Dios, el ser lermento de una comunidad de hombres con espíritu de fratemidad y de gratuidad como hijos de un Padre común; debe ser la caballería ligera que recorre todo el mundo, pero pone su tienda entre los pobres.

Eșa Compañía será así —como lo mucsura la historia reciente- perseguida y crucificada con los pobres de este mundo; será cscándalo para los poderosos, para los ídolos de muertc y sus sacerdotes. Pero así será fiel a su Señor Jesús. Haciendo esto no habrá hecho todo, pero sin hacer esto nada de lo que haga será hoy para la gloria de Dios; haciendo esto encontrará además creatividad para que todos sus trabajos y la cspiritualidad que le es típica sean relevantes y eficaces.

b) Esa espiritualidad teológica descrita en el pártalo anterior en la que se debe concretar la espiritualidad de la Compañía de Jesús ante el enorme desafío del tercer mundo y del mundo de los pobres tiene que cobrar cuerpo en la instilución y en su modo de trabajar.

La Compañía, además de su carisma fundamental, que lo sitúa en la avanzada de la Iglesia que se abre camino por las fronteras de la historia, tiene unas características tradicionales que la capacitan especialemente para responder a este nuevo desafío: 1) es una organización multinacional que está sólidamente establecida tanto en el primer mundo como en el tercer mundo pudiendo convertirse por lo mismo en cadena de transmisión del mensaje de éste sobre aquél; 2) por su forma especial de entender el apostolado y el sacerdocio puede afrontar esta nueva misión que a otras espiritualidades, tradiciones y teologías puede parecer demasiado secular y aun política, cuando de hecho toca a la esencia misma de la evangelización cristiana; 3) cuenta con medios intelectuales e institucionales para convertirse en resonador ante el primer mundo de los gemidos y necesidades del tercer mundo, asi como para hacerse sólidamente presente en todo el tercer mundo donde debería inculturizarse a fondo, de modo que la evangelización y la promoción humana del mismo cobraran el vigor y la novedad que la realidad exige; 4) cuenta con una larga tradición misional que ha realizado espléndidos trabajos, tradición que hoy debería renovarse y mostrarse de nuevo creativa ante el ingente desafío de una inmensa mayor parte de la humanidad que es privada de casi todo mientras unos pocos disfrutan de todo.

c) El Decreto IV de la Congregación General XXXII puso ya a la Compaña en esta nueva línea siguiendo directrices del Vaticano II y de las principales encíclicas sociales. Hoy se trata de dar un paso más y de operativizar todo lo ya propuesio en la línea de la dedicación especial de la Compañia de Jesús a los problemas del tercer mundo, al enfrentamiento none-sur que es más radical cristianamente que el enfrentamiento este-oeste.

Es, desde luego, muy importante el evitar un holocausto nuclear que tendria

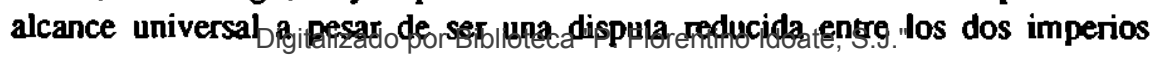


ricos del este y del oeste; en cste sentido, cs importante la lucha por la paz que se han propuesto las iglesias locales del norte. Pero es más importante y más hondo desde el punto de vista de la fe cristiana el buscar solución a la vida de miles de millones de hombres, somctidos a toda suerte de privaciones, opresiones y represiones, de las que son responsables principales los paises del norte y las clases sociales que en cl sur son sus cadcnas de transmisión.

De todos modos no se trata de contraponer las dos tareas, sino de ponerlas en conexión respelando su propia jerarquía; la urgencia de una, que alccla sobre todo a los países del norlc, no debe hacer olvidar la importancia de la otra que afecta casi exclusivamente a los países del sur que representan la mayoria de la humanidad y que ya sulren de hecho no la muerte nuclear, pero sí la agonía permanente de la supervivencia y de la libertad.

d) Evidentemente, la Compañía de Jesús no es una institución capaz por si sola de enfrentar ni siquiera teóricamente el problema norte sur, ni menos de aportar elicazmente a su solución. Pero sí puede realizar una labor significativa y en algún sentido insustituible. Para ello deberia hacer entre otras cosas: 1) analizar cientílica y teológicamente la injusticia que representa el orden actual y el pecado social y personal implicado en la relación diálectica norte-sur; 2) hacer conciencia mundial de este desorden para despertar la conciencia moral especialmente de los hombres del primer mundo; 3) invertir un máximo de recursos en los trabajos del tercer mundo para desde él ayudar a encontrar no sólo un nuevo orden social, sino una nueva civilización de la pobreza, que supere las formas hoy reinantes de capitalismo y socialismo y vayan a la superación de la civilización de la riqueza que se ha convertido en el ideal anti-cristiano de aquellas dos formas económico-políticas; 4) configurar el modo de vida personal e institucional conforme a lo que sería esa civilización de la pobreza y a lo que exige un mínimo de solidaridad con las mayorías pobres de la humanidad, y esto tanto en el primer mundo como sobre todo en el tercer mundo, no dejándose llevar lácilmente por el espejismo de la eficacia; 5) desenmascarar toda suerte de ideologizaciones teológicas, económicas, políticas y culturales que impiden ver la realidad cruda de un orden social, económico, cultural y religioso que ha permitido ver como "natural" y aun querido por Dios el que unos pocos lo tengan todo y los muchos apenas tengan algo mediante mecanismos unas veces sutiles y otras burdos de dominación y de explotación; 6) reajustar la espiritualidad de modo que no se den las dualidades actuales entre intencionalidad y realidad, entre interioridad e institucionalidad, entre comunidad y obra apostólica. Hay una gran connaturalidad y coincidencia entre lo que pide la espiritualidad cristiana, tal como es entendida en los Ejercicios espirituales, y lo que exige la realidad del tercer mundo, el estilo de vida que le corresponde y la acción que se debe realizar.

e) La Compañia debería restructurarse en algunos puntos importantes para Digitalizado por Biblioteca "P. Florentino Idoate, S.J."

Universidad Centroamericana José Simeón Cañas 
ponerse en mejor disposición de adaptarse a la nueva misión: 1) la formación de los jesuilas nuevos y la actualización de los antiguos debería en lo espiritual, en lo intelectual y en lo pastoral acomodarse a este nuevo llamado del Rey eterno, quedando configurada por esa misión que se constituiría en punto esencial de orientación y no en una tarea o en un aspecto más de nuestro apostolado; 2) es importante también la capacitación de todos los jesuitas en esta línea, pero subrayando la necesidad de especializar a muchos de nuestros mejores hombres en esta línea de lo que pudiera llamarse el problema de nuestro tiempo y el desalío mayor de la Iglesia; esta especialización exigiría diferenciación en los estudios y una gran excelencia en el modo de realizarlos; estudios de filosolía, teología, ciencias sociales con especial hincapié en la historia, antropología y política, esudios tecnológicos que enfrenten el difícil problema de la creación y, o apropiación de nuevas tecnologías; 3 ) el traspaso generoso de recursos que hoy se utilizan en el primer mundo, recursos humanos y materiales, lográndose así una comunicación efectiva de bienes que podría resultar muy eficaz, además de representar un signo de la relación justa entre el primer mundo y el tercer mundo; 4) reuniones conjuntas de personas e instituciones que encontrasen modos de colaboración efectiva tras un conocimiento y una experiencia vital del enorme desafío que representa para la conciencia cristiana la existencia del tercer mundo con sus mayorías pobres y explotadas.

6. La Compania de Jesús tiene ante sl, por tanto, un gran desafío, que puede convertirse en llamada a una profunda conversión. Ante el dolor y miseria del mundo, sometido a la tirania del pecado y de su consecuencia la muerte, la Compañla se ve de nuevo convocada por el Padre para ponerse junto a su Hijo con aquellos en que su Hijo ha puesto sus preferencias. A la Compañia le es ofrecida de nuevo la gracia de ponerse con Jesús junto a los más pobres de la tierra para compartir sus dolores y trabajar con ellos para que "venga a nosotros tu reino".

Es un Ilamado que tiene su propia faz histórica. Cuando los grandes sistemas actuales, el capitalismo y el socialismo, no han podido traer al mundo ni la salvación transcedente ni siquiera una liberación histórica a la altura de las necesidades y posibilidades de nuestro tiempo, ha llegado la hora de buscar una solución distinta, que no será un intermedio entre ambos -que no existe-, pero que habra que ir trabajando desde uno u otro de los sistemas, según sean las condiciones de los pueblos y la coyuntura en que se encuentren. Hay que encontrar un sistema que dé vida y no muerte, que dé libertad y no opresión. que dé justicia, que dé paz, solidaridad, comunión. Hay que trabajar por una nueva civilización que no es sin más una civilización de amor, sino que conlleva, como lo anunció Jesús y lo han predicado los mejores de sus seguidores, el ser una civilización de la pobreza en la que nadie tenga más de lo suficiente y en la que a nadie le falte lo necesario. Lo suficiente y lo necesario pueden variar historicamente y culpuralmente pero la unidad fundamental del género humano 
y la condición igual de hijos de Dios y hermanos en Jesucristo no permile diferencias enormes, sobrc todo cuando surgen de la explotación directa o indirecta de unos por otros.

Dedicarse a esta tarea desde los más necesitados y oprimidos, situarse mundialmente en la perspectiva del tercer mundo, pondría a la Compaffla en una tesitura espiritual e histórica que la lanzaría a nuevos logros misionales y a una profunda conversión de su scr aclual. Los resultados ya probados, no exentos de persecución y martirio, que se han dado últimamente cuando se ha situado en esta línea, así lo hacen esperar. 